\title{
The advent of system toxicology: aims and aspect of toxicogenomics
}

\author{
S. M. Neaz Mahmud, Shahin Mahmud, Tamanna Tazrin Tandra, Antora Kar, \\ Elia Jahan Shathy, Nasreen Jahan*
}

\begin{abstract}
Department of Biotechnology and Genetic Engineering, Mawlana Bhashani Science and Technology University, Santosh, Tangail-1902, Bangladesh
\end{abstract}

Received: 09 June 2016

Accepted: 04 July 2016

\section{*Correspondence to:}

Nasreen Jahan,

Email: naza.bge@gmail.com

Copyright: () the author(s), publisher and licensee Medip Academy. This is an openaccess article distributed under the terms of the Creative Commons Attribution NonCommercial License, which permits unrestricted noncommercial use, distribution, and reproduction in any medium, provided the original work is properly cited.

\begin{abstract}
Last fifty years a significant advancement has been established in biological science. It happened due to the discovery of gene, genome and genetic code, function of genes and mutation of genes. Through this, the scientists have discovered that genetic code is the building block and fundamental of all molecular activity in biological system. According to this, several molecular techniques have been established to prove molecular events, effects of chemical exposure within individuals and environment. For this evaluation, the necessary of toxicogenomics is crucial, that deals with the effects of chemical in changing the genetic pattern along with mutation into gene. Toxicogenomics also deals with transcription of proteins and metabolite profiling to investigate the interaction of genes and environment stress in disease. Toxicogenomics also described the altered expression of genes caused by mutation and chemical exposure that cause several disease and show toxicant functions in cell. The main objective of toxicogenomics is to remove this exposure and provide remedy of these toxical diseases. The use, application, correlation, combination and collaboration of different significant, major, modern biological fields like proteomics, transcriptomics, bioinformatics, microarray and several other molecular process is carried out by toxicogenomics that gradually evolving in systems toxicology. This review recovered the evolution and significant application of the different fields of toxicogenomics.
\end{abstract}

Keywords: Toxicogenomics, System toxicology, Proteomics, Genomics, Metabolomics

\section{INTRODUCTION}

Toxicogenomics is an important era of genetics that was first coined/ invented in 1999 which outlines the relationship between genomics and toxicology. ${ }^{1}$ After 19th century the period of toxicogenomics has rapidly shown its flow of growth. For few toxicological screening there is a method called microarray that can analyse both genome sequencing and expression of gene thus toxicology has become an advanced tool for toxicological screening, more complex and complicated discovery of toxicity, using pattern of chemicals, drugs and measurement of their safety and it also contributed in discovery of new drugs. ${ }^{2,3}$ In order to understand and rearrange the genome now a days toxicogenomic research is used by the scientists as a tool that can make possible the study of unlimited data sets from microarray in which new approaches and methods can be required. The latest genomic technology that seems to be incomprehensible provides information to understand the incorporation of toxicological process which is the intent goal of toxicogenomic research. ${ }^{4}$

The study of stressors and their scathe on living organisms and their environment can be defined as the main aspect of toxicogenomic research. There are different portions of toxicology one of which negotiates with risk assessment, identification of hazards and toxicological mechanistic. Another portion of toxicology can help in understanding the mode of action of different types of drugs and chemicals and also can make the advancement of the efficiency of their risk assessment.

To reduce toxicity toxicogenomics also can be used in hazard screening, mechanistic information, exposure assessment and developmental exposure. Different 
chemical and pharmaceutical compounds developed by different industries needs to monitor and discern and such can accelerate the effects of toxicogenomic technique on those compounds. The screening method needs something that can offer new drug's potential adverse potential outcomes. This allows the safety evolution of those compounds's intelligent advancement into late stages. ${ }^{5}$

Using toxicogenomic study a rich genomic library can be made using data from various investigative programs. This genomic library can be used to understand responses of different cell and organs against toxic particles. In the progression of this process proteomics, transcriptomics and metabonomics have some influences. The application of gene expression for understanding the environmental stressor effects on biological system has increased with quick development in genomic, proteomic metabonomic based technologies. ${ }^{6-15}$ This technologies can be applied on protein, mRNA and metabolite analysis to study hazard effects on organisms. ${ }^{16-22}$

The new field of toxicogenomics is the main topic of this review and also depictures some of its achievements and research approaches. This process also will show the data integration process derived from proteomics, transcriptomics and metabonomics and also about the advent of system toxicology. ${ }^{23-29}$ All the interactions elements in a given biological system under toxicant perturbation to attain a mechanistic understanding of toxical response has tried to discuss with system toxicology as with system biology. ${ }^{30}$

\section{The evolution of molecular toxicology and toxicogenomics}

With the discovery of DNA structure by Watson and Crick the history of molecular biology has been restrained. The capacity of fully translating the codes to function is really challenging for nearly 60 years. The factual progression of molecular biology to toxicology provides for translating molecular concern to organism health. Southern blotting is the method of visualization of genetic materials in a manner of feasible that has been established in 1975. Then the modified version of southern blotting has been established as northern blotting that detects RNA transcript. ${ }^{31}$ Thus a technological breakthrough occurred which qualified the toxicologists to measure and track the changes within the DNA transcript and detects the changes in gene expression following the exposure of cells to toxicants. The first application of northern blotting was prepared to determine lactate dehydrogenase transcript following exposure to materials. ${ }^{32,33}$ Now there are more rapid and higher output methods has been introduced that needs a very small amount of sample and can track the molecular events in genomic level. Microarray chip is the most advanced technology for such kind of measurement published first in mid-1990's. DNA microarray has two types of functions, one which was produced with "on- chip synthesis" of short oligo sequences carry technologies from semiconductor industry. ${ }^{34}$ The purification of longer length DNA "spots" generated by PCR is the next function of microarray chip. ${ }^{35,36}$ The result for either platform was a very simple array that could uniquely permit the probing the whole DNA transcript profile and test the expression of host's functional genes for any biological hybridized RNA and it provided a new field in toxicogenomics. In present "toxicogenomics" refers the interface of several functional genomic approaches that are used to understand the mood of toxicity. ${ }^{37}$ Not only National Academy of Sciences but also the leading scientists also provided the need and limitations of toxicology towards calculation of individual susceptibility and risk, measurement of exposure, elucidation of mechanism. ${ }^{38}$ Fruitful execution of microarray technology needs the development of mutual science across several rules such as chemistry, engineering, mathematics, computer science, molecular biology and engineering. ${ }^{39}$ In all areas of biology many papers have been published about summery of micro array technology. Even there are more than 500 review papers and illustrating the concepts of toxicogenomics.

\section{The evolution of the field of toxicogenomics}

\section{Toxicogenomics: aims and methods}

Toxicogenomics aims three principal goals: it implies the relationship between environmental stress and human disease susceptibility, it identify useful biomarkers of disease and exposure to toxic substances; and elucidate the molecular mechanisms of toxicity. ${ }^{40}$ A typical toxicogenomics study might involve an animal experiment. This experiment includes three treatment groups: high dose and low-dose treatment groups and a vehicle control group that has received only the solvent used with the test agent. These groups are experimentally observed at two or three points in time, which are done three to five animal subjects per group. So a toxicogenomics study implies a simple, acute-toxicity study. ${ }^{41}$ These two groups are differ in their scope of the response from which they are detected and in the methods used. The high-dose treatment are expressed with toxic response, the toxicogenomics research can identify this toxic response using the global-measurement techniques. In general toxicogenomics study, a list for each sample genes that are expressed differentially are created. If it is not possible the profile-analysis methods are applied to dose-related and time course studies for the identification of genes and gene profile of sample organism. ${ }^{41}$ By using the knowledge that is extracted and assembled from literature analysis a comparative analysis and modeling of molecular-expression datasets is created and within this database it is possible to differentiate the adaptive responses of biological systems. ${ }^{42}$ It also deals with those changes that are associated with clinical or visible adverse effects. Last 5 years, the field of toxicogenomics has increased mostly, within the concept 
of gene-expression profiles as 'signatures' of toxicant classes, disease subtypes or other biological endpoints. Through an analytical research these signatures are designed for predictive biomarkers of toxicant effects and this signature are now contribute to understand the dynamic alterations in molecular mechanisms that are associated with toxic and adaptive responses. Now the research work related to a toxicogenomics study are increasing largely and the amount of gene-expression database also increase. For the examination of per dosetime group, every animal tissue requires 18-45 microarrays and 2000 or more transcripts per array required for attendant measurement. ${ }^{43}$ Also, every animal contain treatment-associated data on their total body and organ-weight measurements, clinical chemistry measurements and microscopic histopathology findings for several tissues. ${ }^{44-46}$ This important data collection, management and integration are done carefully and this process is very crucial for the experimental protocol and for interpreting toxicological outcomes. So, all this data must be collected in terms of dose, time and severity of the toxicological or histopathological phenotype. This experimental data are compiled to analyse with toxicoinformatics tools and computational modelling and it derive a new important understanding of toxicantrelated disease. ${ }^{47}$ Toxicogenomics is now integrating the multiple data collection derived from transcriptomics, proteomics and metabonomics with traditional toxicological and histopathological endpoint-evaluation. This integration has the potential to develop our understanding on the relationship between toxicological outcomes and molecular genetics. Now toxicology and toxicogenomics are developed predominantly on individual chemicals and stressors into a knowledgebased science. ${ }^{40}$ Toxicogenomics stands by 'predictive toxicology that is the phenotypic responses of an agent on a related strains and this can be used to identify the mode of action of same molecule in related strain or other species. This result also used for other toxicogenomics researches related to genotypes and the species are assimilated for multi-genome knowledgebase. This knowledgebase are find out by chemical formula or stressor type, by gene, protein or by analysing the metabolism of metabolic molecule or by phenotypic outcome of other entities that express the result of newly tested agent. $^{48,49}$ All this things make toxicology become an information science and the genomics, proteomics are also helped by this (Figure 1). Example of toxicogenomics flow scheme. In this example, individual rodents are exposed to varied doses of compound and tissues are collected at various time points and subject to microarray analysis. Calculations are made to (1) determine the significantly altered genes in each sample and (2) map these gene changes into annotated pathways. This allows for initial assessment of a view to potential mechanisms of tissue response to compound perturbation. As illustrated by (3), expression files may also be mapped against archived files to determine similarity of compound action/response to other compounds that have been previously studied in the database. It should be noted that analyses may be conducted on individual dose/time profiles or across dose and time response with an assessment of "trend.",39

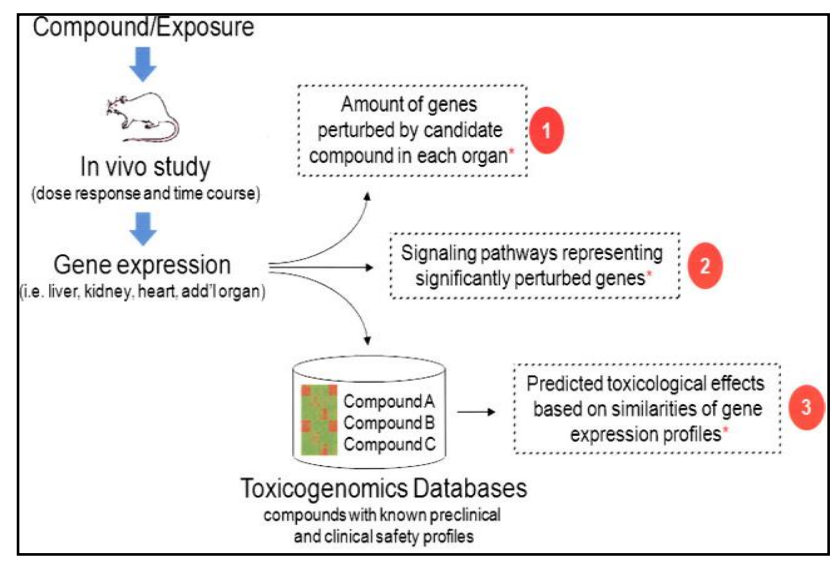

Figure 1: Analysis can be performed at each dose/time point/organ independently (characterizing effects based on the dose level of the compound).

\section{Use of toxicogenomics in risk assessment}

The regulatory agencies increase efforts to incorporate toxicogenomic data into risk assessment. These data are prepared ago to replace existing required testing regimens in risk assessment and regulatory toxicology. Toxicogenomic technologies will be further developed to increase capabilities in the following sectors:

\section{Exposure assessment}

Modern toxicogenomic information is more discriminating, predictive, and sensitive that currently used to evaluate toxic exposure and associated with exposure to individual chemicals and to chemical mixtures.

\section{Variability in susceptibility}

The genetic contribution in susceptibility to human variation by toxic effects of pharmaceuticals and this toxic effect varies from person to person. Gene - gene interactions are another important area of research for understanding human susceptibility and assess differences in susceptibility in larger populations. ${ }^{38}$

\section{Cross-species extrapolation}

Despite the dependence on animal models in toxicologic research for predicting human health effects in the regulatory area, there are important differences between how animals and humans respond to different chemicals. ${ }^{50}$ Otherwise, there are limitations for analysis of emerging data - rich approaches such as- genotyping, mRNA analysis, protein analysis and metabolite analysis. $^{51}$ 


\section{Hazard screening}

Hazard screening can be comprehensive, intended to detect all potential hazards, or it can be more limited, detecting only a specific type of hazard. Toxicogenomic screening methods depends greatly on the setting. It should be integrated into applicable chemistry regulatory and safety programs. ${ }^{52}$

\section{Mechanistic information}

Toxicogenomics holds the promise of providing new insights into molecular mechanisms of a variety of toxicities. Chemical-specific patterns of altered gene expression can be revealed using high - density microarray analysis.

\section{Dose-response relationships}

Toxicogenomics has the potential to improve understanding of dose-response relationships, particularly at low doses and attention must focus on characterizing toxicogenomics responses at low doses. ${ }^{53,54}$

\section{Developmental exposures}

Exposure of developing tissues or organs to an adverse stimulus or insult during critical periods of development is occured. Toxicogenomic technologies are expected to reveal more efficient to detect the potential effects of exposure to toxic substances in early development stage. ${ }^{55,56}$

\section{Data integration}

The goal of toxicogenomics is to integrate data from different studies and investigative platforms to create a richer and biologically more refined understanding of the toxicological response of a cell, organ or organism. For example, one goal would be describe the interplay between protein function and gene expression, or between the activity of certain metabolizing enzymes and the excretion into serum or urine of populations of small metabolites. The integration of data from different domains such as proteomics and transcriptomics or transcriptomics and metabolomics has been reported. In these experiments, tissue samples that were resulting from the same individual animals or from comparably treated animals were analysed in parallel using different technologies. ${ }^{57-59}$ However, the data from different studies were integrated only after a shortlist of differentially responsive transcripts or protein spots had been derived. The experience gained from integrating globalproteomics or metabolomics data, such as spot intensities from 2D gels or metabolomics fingerprint data from NMR, tells us that cluster or principal component analysis can be done to derive global signatures of molecular expression in much the same way as in transcriptomics analyses. $^{60}$ If biological samples segregate into unique clusters that show similar expression characteristics, further efforts can be made to distinguish the new proteins or metabolites that are expressed in these samples. ${ }^{40,61}$ Further steps can also be taken to calculate these proteins or metabolites as potential biomarkers and as a means to determine the underlying toxicological response. Although software is plentiful for managing expression- profiling data at the laboratory level, there is a compelling need for public databases that combine profile data with associated biological, chemical and toxicological endpoints. ${ }^{62}$ Comparisons of gene, protein and metabolite data in public databases will be valuable for promoting a global understanding of how biological systems function and respond to environmental stressors. ${ }^{44}$ As these repositories are developed, experiments will be deposited from disparate sources, using different experimental designs, but targeting the same toxicity endpoint or a similar class of toxicant. ${ }^{63}$ In these cases, it will be important that the databases integrate data from related studies before data mining occurs. To maximize the value of deposited datasets, the repositories must also be able to integrate data from different technological domains. ${ }^{64}$ Furthermore, a standard representation of data types in each domain is a pre-requisite for efficient and accurate storage, access, analysis, comparison and data exchange. International standards that encompass technological and biological domains are under development by the microarray gene expression data (MGED) society, or reporting structure for biological investigations (RSBI), working group. ${ }^{65,66}$ Furthermore, members of regulatory bodies are working with scientists from industry, academic and government laboratories participating in the ILSI genomics committee and clinical data interchange standards consortium to develop standards for the exchange, analysis and interpretation of transcriptomics data.

Some steps have been taken to extend toxicogenomics and combine it with computational approaches such as pharmacodynamics and pharmacokinetics. ${ }^{30}$ These two systems can be used to derive quantitative estimates of the dose of the test agent or its metabolites that are present in the specific tissue at any time after treatment. Thereby allowing molecular expression profiles to be anchored to internal dose, as well as to the time of exposure and to the toxicant-induced phenotype. ${ }^{43}$ Relationships between gene, protein and metabolite expression can then be described both as a function of the applied dose of an agent and the ensuing kinetic and dynamic dose-response behaviours that occur in various tissue compartments. Such models also must take into account the fact that the transcriptome, proteome and metabolome are themselves dynamic systems, and are therefore subject to significant environmental influences, such as time of day and diet. ${ }^{67}$

Despite the numerous successes of toxicogenomics in the context of toxicology, a poorly addressed but confounding issue that is pertinent to drug safety and human risk assessment is the effect of the individual 
genetic background on the response of an animal or human patient. ${ }^{68}$ The PharmGKB pharmacogenetics knowledgebase catalogues the relationship between different human genetic backgrounds and susceptibility to drug therapy. In addition, the NIEHS environmental genome project is identifying SNPs in genes that are important in environmental disease, detoxification and repair. ${ }^{69}$ Linking toxicogenomics knowledge bases with those containing information about SNPs and human susceptibility will gradually lead to a more complete picture of the relevance of the responses and genotypes of surrogate animal species to human risk assessment.

\section{Microarray analysis}

The detection of expression level of thousands of particular genes of different biological samples is possible by the use of DNA chips, or microarrays, quantitative analysis. This process shows the differences between individual cells with diseased cell. Microarrays are constructed by immobilization of DNA sequences, the coding sequence of genes of interest are bind to a solid support such as glass slide or nylon membrane. ${ }^{70} \mathrm{~A}$ mRNA are prepared from the labelled tissue and then hybridized in the form of reverse transcribed cDNA microarray. This mRNA is visualized using phosphorimager scanning or other process. ${ }^{71}$ Then further analysis is done using appropriate software, which allows determination of the extent of hybridization of the labeled probes to the corresponding arrayed cDNA spots, and provides a comparison between controls with test samples that permits quantitative analysis of changes in gene expression associated with treatment. ${ }^{61}$

There are many commercial microarrays are now available, varying from those comprising several hundred genes (usually immobilized on a nylon membrane and probed with radiolabeled cDNA) to those harborin genes of thousands of oligonucleotide sequences (immobilized on glass slides and analysed using dual fluorescent- probe technology). ${ }^{72}$ These arrays can use either "broad spectrum" or custom designed to profile particular tissues, biological pathways, or even disease states. In this regard, microarrays designed to profile genes involved in response to toxic insult have been developed by commercial vendors, pharmaceutical companies, and academic institutions. ${ }^{1}$

\section{Challenges and technical considerations}

From recent analysis three general challenges have been predict in human health risks from chemical stressors raises: the side effects of thousands of chemicals and other stressors that are present in the environment, the time and dose parameters that define the relationship between exposure to a chemical and disease, and the genetic and physiological diversity of human populations and of organisms used as surrogates to determine the adverse effects of a toxicant. This knowledge and genetics can help us to understand environmentally induced diseases, assess risk and increase awareness of public-health. Associated with these challenges, the technical issues are described below:

\section{Technical issues}

The alteration in genome sequence cause alteration in mRNA, protein and metabolic concentration of tissue extract are used as 'signature' that identifies the gene changes. It also identify several gene products that are involved in a toxic process depends on specific cell types in which the target-gene transcripts and products are located. Northern or western blotting, real time PCR, are generally used to verify the expression profile of a gene or to selectively analyse its expression as a function of toxicant dose or time of exposure. ${ }^{73,74}$ In vivo hybridization, immunohistochemistry and other techniques can be used to identify the specific cell types that maintain the gene expression. ${ }^{75}$ Within this method most of the molecular expression of cell can be analysed. Only a limited number of cells require cell separation methods that limit the contribution of cell type to gene expression. $^{76}$

Toxicogenomic analysis deals with biological sample and the most used biological sample contain many cell types, especially the nacrotic tissue. Laser capture microdissection (LCM) provide more homogenous samples, this method isolates individual cells or sections of tissue from a fixed sample. ${ }^{77}$ The use of LCM minimizes the contributions of non-target cell populations in comparisons of diseased and normal tissue, but also introduces handling and preparation steps that can affect detection accuracy. The modern technology also takes part in cell sampling, it also have the ability to accurately detect signals from smaller samples. For example, it will frequently be used to amplify mRNA from the biological sample used for transcriptomics analysis. It is very important to detect weak signals or small but biologically important changes in expression levels, because the toxicologists explore the initial steps in biologicalsignalling cascades and compensatory processes. Now strong and small signals can be detect by cDNA microarray hybridization technique. This technique also suitable for identifying signals within a mixed cell population in samples that are diluted by up to 20fold. $^{75,78}$ Also this technology can probably detect a strong signal from a population comprising 5-10\% of the total tissue. But this technology have some limitation, it miss some subtle changes associated with signalling or other responses to a stressor. The main purpose of this method is to identify the genomic changes that occur into a cell. Another tool for identifying the toxicant effects is mRNA analysis, protein structure analysis, modification and global protein-expression provides distinct advantages for understanding the functional state of the cell or tissue. ${ }^{79}$ New methods are applied to profile the capacity of proteins with antibody arrays and surfaceenhanced laser-desorption mass spectrometry. New insights into the function of genes are accessible by 
measuring the alter patterns of mRNA and protein expression in accessible tissues such as serum. ${ }^{8}$ This might help to functioning of genes in the context of toxicity and guide the search for protein biomarkers of toxicant exposure or predictive toxicity.

\section{Bioinformatics challenges}

To understand or realize all the molecular mechanism in toxicogenomics, an extensive investment in bioinformatics is required. It will increase the number of molecular research that enhances biological sense from the many of interrelated numerical-molecular identifiers and their associated explanation. Bioinformatics depends on biological and mathematical modelling. Bioinformatics and mathematical modelling tools provide powerful approaches for identifying the patterns of biological response that are placed in genomic datasets. A global molecular database has been established through bioinformatics form genomics or proteomics technology and is currently controlled by 'bioinformatics bottleneck'. The identification process of genes, proteins and metabolites are improved by bioinformatics and it opens the field of toxicogenomics in high-throughput applications in drug development and toxicant evaluation. $^{80}$ Several annotation problems from useful resources address by linking identifiers used in genomic databases at the National Centre for Biotechnology Information (NCBI), the European Molecular Biology Laboratory (EMBL) and the DNA Data Bank of Japan (DDBJ) to other annotation resources. ${ }^{81}$ Annotation inconsistencies is the knowledge of the sequence of the actual nucleotide or protein that is used to query the genome. Now more and more advanced bioinformatics tools are used to extract information from microarray results. These tools provide a valuable result when the data used have a high degree of internal specificity and accuracy. ${ }^{82}$ Bioinformatics link between biological profile and statistical analysis and this improve the bioinformatics experimental knowledgebase. ${ }^{44}$ This means that once a set of genes with altered expression is identified, their biological functions must be ascertained. Mechanical interpretation of transcript changes provides non-standard or imprecise annotation of a sequence element. The comparison of results might be hampered by the difference in annotation within and among diverse microarray platforms. This contradiction are arises from annotation resources that use different lexicons, or from annotation information being compiled at different times. Further bioinformatics and interpretive challenges arise at many levels of biological organization. ${ }^{83}$ Now our levels of understanding about the global molecular-landscape are limited only the lower levels of complexity (genes/proteins, gene/protein groups, and biological pathways). Now our knowledgebase on bioinformatics might be termed linear toxicoinformatics; that deals with the environmental stimuli and responses, over dose and time, following a toxicological stress. Within this research the toxicologists and risk assessors' advice some key events and linear modes-of-action for environmental chemicals and drugs. ${ }^{84}$ On the other hand, environmental stimuli change the highly nonlinear cellular-expression state. Therefore, the statistical and bioinformatics-based separation of the complex adaptive, pharmacological and toxicological responses of drugs, chemicals and even dietary constituents will probably be a matter of degree reflecting the kinetic and dynamic responses of specific tissues to toxicants as directed by the genome, the genetic heritage of the individual and that individual's current and prior exposures. ${ }^{85}$

\section{Systems toxicology}

Ideker used the phrase 'systems biology' to describe the integrated study of biological systems at the molecular level - involving perturbation of systems, monitoring molecular expression, integrating response data and modeling the systems' molecular structure and network function. ${ }^{86}$ Here, we similarly use the phrase 'systems toxicology' to describe the toxicogenomics evaluation of biological systems, involving perturbation by toxicants and stressors, monitoring molecular expression and conventional toxicological parameters, and iteratively integrating response data to model the toxicological system. $^{87,88}$

Several approaches are being developed to model network behaviour, with different assumptions, data requirements and goals. However, it is not likely that toxicogenomics and systems toxicology models will be assembled exclusively from knowledge of cellular components, without equivalent knowledge of the response of these components to toxicants. ${ }^{89}$ Therefore, the 'stress testing' of the structural biology of the system and the capture of that data in the context of the functioning organism adapting, surviving or succumbing to the stress will be required. ${ }^{90}$ Development of a knowledgebase to accurately reflect network-level molecular expression and to facilitate a systems-level biological interpretation requires a new model of data management, data integration and computational modeling. A knowledgebase that fully embraces systems toxicology will use precise sequence data to define macromolecules, interaction data based experimentally on co-localization, co-expression and analyses of proteinprotein interactions, and functional and phenotypic data that is based on gene knockouts, knockins and RNAinterference studies, in addition to studies of responses to chemical, physical and biological stressors. ${ }^{53,91}$ These data will allow specific molecules to be accurately related to the biological phenomena that reflect both the normal and the stressed cell, tissue, organ or organism. In the best circumstances, a system toxicology approach will build a toxicogenomics understanding from global molecular-expression changes that are informed by PBPK or PD modelling and biologically-based dose-response (BBDR) modelling. The challenge in constructing a robust systems toxicology knowledgebase is formidable. 


\section{Toxicogenomics in future year}

The power and potential of new toxicogenomics methods revolutionize toxicology. Technological improvement brought out a new era in toxicological science that are already in use allow RNA profiling of formalin-fixed tissues, tissue archived from generations to generations of toxicological studies make possible the gene-expression analysis. Several methods that are used to array hundreds of toxicologically relevant protein antibodies, and to profile hundreds of small molecules in high-throughput mode using gas chromatography, liquid chromatography or mass spectrometry are developed gradually. ${ }^{92}$ Toxicoproteomics research achievements are now used in identification, measurement and evaluation of proteins and other biomarkers that are more accurate, sensitive and specific than those are available now, and they target only particular human genetic subpopulations. Identifying alterations in the levels of small endogenous molecules as important changes in a sequence of key metabolic events is possible by metabolomics, now this 'metabolite fingerprints are used to diagnose and define the ways of specific chemicals, environmental exposures or stressors cause disease. ${ }^{93}$ This process also has the ability to detect damage to particular organs by screening alterations in serum and urine compounds and it is expected the more sensitive detection of exposure or risk factors. ${ }^{94} \mathrm{~T}$ oxicogenomics also response to environmental exposures are related to individual genotype, lifestyle, age and exposure history. ${ }^{43}$ Toxicogenomics will help to keep balance of these factors that influence the stability between healthy and disease states. Environmentally realistic dose-regimens and therapeutic global observation of genomic response can increase the significance of toxicogenomics. It will help to delineate the modes-of-action of various classes of agents and the unique genetic attributes of certain species and population subgroups that render them susceptible to toxicants. Various strains of a species that are sensitive or resistant to the chemical induction of specific disease phenotypes, studying there genomics will be particularly valuable. ${ }^{30}$ Using the genomic knowledge to the phylogenetic analysis of both core, conserved biological processes and to the toxicological responses seen in different species that will provide a comparative insight into genetic susceptibility and probable disease outcomes. ${ }^{95}$ Our overall understanding of mechanisms of toxicity and disease etiology as integrated toxicogenomics databases are developed by the combined application of genomics technologies. Integration of data on gene, protein or metabolite changes collected in the respect of dose, time, target tissue and phenotypic severity across a range of species from yeast, to nematode, to man will provide the comparative information needed to assess the genetic and molecular basis of gene. ${ }^{30}$ Now we consider toxicology as an information science that will facilitate scientific discovery around all biological species, chemicals and disease outcomes. Although development of public toxicogenomic data repositories are great challenge for scientist, the nucleotide-sequence databases GenBank, EMBL and DDBJ - are examples of the benefits of sharing data to the wider scientific and medical community. ${ }^{96}$ The remarkable improvement in bioinformatics methods and data mining tools also develops the toxicogenomics database and also increase the number of researchers who are working on them. ${ }^{82}$ We believe that a predictive systems toxicology will gradually evolve, aided by knowledge that is systematically generated through literature mining comparative analysis and iterative biological modelling of molecular-expression datasets over time. ${ }^{42}$ However, the development of a comprehensive and public knowledgebase that improve the era of toxicology and environmental health and this improvement brings by the vast numbers and diversity of drugs, chemicals and environmental agents, and the diversity of species in which they act. The NCT is working restlessly to create the chemical effects in biological systems (CEBS) knowledgebase a public resource and its ultimate goal is to enable health scientists to understand and mitigate or prevent adverse environmental exposures and related diseases in the twenty-first century. ${ }^{97}$

\section{Summary}

Now toxicogenomics are moving forward very quickly. If we admire the development of toxicogenomics, we noticed that it is standing still, on the other hand it advancing quickly. This time toxicogenomics have taken a lot of challenge, for this competition the actual field of toxicogenomics are expressed largely. This process also ensures its future progress. If the progress will continue in this rate, it will reach in a milestone. Toxicogenomics research aims to formation of genomic database and it can be opined that this process will be finished within 5 years and it will be a breakthrough achievement of this field. ${ }^{98}$ The biologist find lots of information from this database and this process will continue to update. Next 10 to 20 years toxicogeomics make progress and establish a uniform technical measure and definition of gene expression. ${ }^{99-100}$ It brings a remarkable success in the field of discovery and validation of toxicities and disease. Technical development also makes it easy and will enable simultaneous probing of genetic, genomic, proteomic, and metabolomics events. The data obtained from toxicogenomics biomarker are highly used to regulate environment to better inform the risk assessment from invivo and in-vitro system. ${ }^{101}$ This modified test has now minimize animal testing and also helps to create appropriate model from human in-vitro based assays and provide a way to predict insilico models that will help to reduce animal use and cost of experiments conducted hazard and risk.

Funding: No funding sources Conflict of interest: None declared Ethical approval: None required 


\section{REFERENCES}

1. Nuwaysir EF, Bittner M, Trent J, Barrett JC, Afshari CA. Microarrays and toxicology: The advent of toxicogenomics. Mol Carcinog. 1999;24:153-9.

2. Hayes KR, Bradfield CA. Advances in toxicogenomics. Chem Res Toxicol. 2005;18:403-14.

3. Hamadeh HK, Todd M, Healy L, Meyer JT, Kwok AM, Higgins M, et al. Application of genomics for identification of systemic toxicity triggers associated with VEGF-R inhibitors. Chem. Res. Toxicol. 2010;23:1025-33.

4. Cunningham1 ML. Toxicological highlight putting the fun into functional toxicogenomics. Toxicological Sciences. 2006;92(2):347-8.

5. Hamadeh HK, Amin RP, Paules RS, Afshari CA. An overview of toxicogenomics. Curr Issues Mol Biol 2002;4:45-56.

6. DeRisi J, Penland L, Brown PO, Bittner ML, Meltzer PS, Ray M, et al. Use of a cDNA microarray to analyse gene expression patterns in human cancer. Nat Genet. 1996;14:457-60.

7. Duggan DJ, Bittner ML, Chen Y, Meltzer PS, Trent JM. Expression profiling using cDNA microarrays. Nat Genet. 1999;21:10-4.

8. Huang Q, Dunn RT, Jayadev S, DiSorbo O, Pack FD, Farr SB, et al. Assessment of cisplatin-induced nephrotoxicity by microarray technology. Toxicol. Sci. 2001;63:196-207.

9. Lueking A, Horn M, Eickhoff H, Bussow K, Lehrach $\mathrm{H}$, Walter $\mathrm{G}$. Protein microarrays for gene expression and antibody screening. Anal Biochem. 1999;270:103-11.

10. Page MJ, Amess B, Rohlff C, Stubberfield C, Parekh R. Proteomics: a major new technology for the drug discovery process. Drug Discov Today. 1999;4:5562.

11. Rubin RB, Merchant M. A rapid protein profiling system that speeds study of cancer and other diseases. Am Clin Lab. 2000;19:28-9.

12. Steiner S, Anderson NL. Pharmaceutical proteomics. Ann NY Acad Sci. 2000;919:48-51.

13. Weinberger SR, Morris TS, Pawlak M. Recent trends in protein biochip technology. Pharmacogenomics. 2000;1:395-416.

14. Richard A, Yang C, Judson R. Toxicity data informatics: supporting a new paradigm for toxicity prediction. Tox Mech Meth. 2008;18:103-18.

15. Martin MT, Mendez E, Corum DG, Judson RS, Kavlock RJ, Rotroff DM, et al. Profiling the reproductive toxicity of chemicals from multigeneration studies in the Toxicity Reference Database (ToxRefDB). Toxicol Sci. 2009;110:18190.

16. Afshari CA, Nuwaysir EF, Barrett JC. Application of complementary DNA microarray technology to carcinogen identification, toxicology, and drug safety evaluation. Cancer Res. 1999;59:4759-60.

17. Farr SRND. Concise review: gene expression applied to toxicology. Toxicol Sci. 1999;50:1-9.
18. Henry CM. DNA microarrays in toxicology. Anal Chem. 1999;71:462A-464A.

19. Rockett JC, Dix DJ. Application of DNA arrays to toxicology. Environmental Health Perspectives. 1999;107:681-5.

20. Hamadeh H, Afshari CA. Gene chips and functional genomics. American Scientist. 2000;88:508-15.

21. Pennie WD, Tugwood JD, Oliver GJ, Kimber I. The principles and practice of toxigenomics: applications and opportunities. Toxicol Sci. 2000;54:277-83.

22. Rockett JC, Dix DJ. DNA arrays: technology, options and toxicological applications. Xenobiotica. 2000;30:155-77.

23. Hooker TM. The Brave New World of Toxicogenomics. Environmental Compliance Litigation; 2001:16-5

24. Iannaccone PM. Toxicogenomics: the call of the wild chip. Environ Health Perspectives. 2001;109:A8A11.

25. Olden KG. Genomics: implications for toxicology. Mutation Res. 2001;473:3-10.

26. Smith LL. Key challenges for toxicologists in the $21^{\text {st }}$ century. Trends Pharmacol Sciences. 2001;22:281-5.

27. Tennant RW. The National center for toxicogenomics: using new technologies to inform mechanistic toxicology. Environ. Health Perspect. 2002;110:A8-A10.

28. Hamadeh HK, Bushel P, Paules RS, Afshari CA. Discovery in toxicology: Mediation by gene expression array technology. J Biochem Mol Toxicol. 2001;15:231-42.

29. Hamadeh HK, Nuwaysir EF, Paules RS, Barrett JC, Afshari CA. cDNA microarray technology: Merging toxicology and genomics. Comments Toxicol; 2002. Available http://citeseerx.ist.psu.edu/viewdoc/download?doi=1 0.1.1.113.9327\&rep=rep1\&type=pdf.

30. Waters MD. Systems toxicology and the chemical effects in biological systems knowledge base. Environ Health Perspect. 2003;111:811-24.

31. Alwine JC, Kemp DJ, Stark GR. Method for detection of specific RNAs in agarose gels by transfer to diazobenzyloxymethyl-paper and hybridization with DNA probes. Proc Natl Acad Sci. U.S.A. 1977;74:5350-4.

32. Miles MF, Hung P, Jungmann RA. Cyclic AMP regulation of lactate dehydrogenase. Quantitation of lactate dehydrogenase M-subunit messenger RNA in isoproterenol-and N6, O2'-dibutyryl cyclic AMPstimulated rat C6 glioma cells by hybridization analysis using a cloned cDNA probe. J Biol Chem. 1981;256:12545-52.

33. Huang RP. Detection of multiple proteins in an antibodybased protein microarray system. J Immunol Methods. 2001;255:1-13.

34. Chee M, Yang R, Hubbell E, Berno A, Huang XC, Stern D, et al. Accessing genetic information with high-density DNA arrays. Science. 1996;274:610-4.

35. Hughes TR, Marton MJ, Jones AR, Roberts CJ, Stoughton R, Armour CD, et al. Functional discovery 
via a compendium of expression profiles. Cell. 2000;102:109-26.

36. Schena M, Shalon D, Davis RW, Brown PO. Quantitative monitoring of gene expression patterns with a complementary DNA microarray. Science. 1995;270:467-70.

37. Brown PO, Botstein D. Exploring the new world of the genome with DNA microarrays. Nat Genet. 1999;21(Suppl 1):33-7.

38. National Research Council. Committee on applications of toxicogenomics technologies to predictive toxicology, "applications of toxicogenomic technologies to predictive toxicology and risk assessment. National Academies Press, Washington DC; 2007.

39. Afshari CA, Hamadeh HK, Bushel $\dagger$ PR. The evolution of bioinformatics in toxicology:advancing toxicogenomics. Toxicological Sciences. 2011;120(S1):S225-S237.

40. Fostel WJM. Toxicogenomics and systems toxicology: aims and prospects, Nature Reviews; 2004:5.

41. Hamadeh HK. Methapyrilene toxicity: anchorage of pathologic observations to gene expression alterations. Toxicol Pathol. 2002;30:470-82.

42. Zweiger G. Knowledge discovery in geneexpressionmicroarray data: mining the information output of the genome. Trends Biotechnol. 1999;17:429-36.

43. Kaput J, Rodriguez RL. Nutritional genomics: the next frontier in the postgenomic era. Physiol Genomics. 2004;16:166-77.

44. Mattes WB, Pettit SD, Sansone SA, Bushel PR, Waters MD. Database development in toxicogenomics: issues and efforts. Environ Health Perspect. 2004;112:495-505.

45. Xiao HS, Huang QH, Zhang FX, Bao L, Lu YJ, Guo $\mathrm{C}$, et al. Identification of gene expression profile of dorsal root ganglion in the rat peripheral axotomy model of neuropathic pain. Proc Natl Acad Sci USA. 2002;99(12):8360-5.

46. Stoeckert CJ, Parkinson H. The MGED ontology: a framework for describing functional genomics experiments. Comp Funct Genom. 2003;4:127-32.

47. Tennant RW. The national center for toxicogenomics: using new technologies to inform mechanistic toxicology. Environ Health Perspectives. 2002;110(1):A8-10.

48. Morgan KT, Pino M, Crosby LM, Wang M, Elston TC, Jayyosi Z, et al. Complementary roles for toxicologic pathology and mathematics in toxicogenomics, with special reference to data interpretation and oscillatory dynamics. Toxicol. Pathol. 2004;32(Suppl 1):13-25.

49. Tsai YJ, Lin CT, Huang CT, Wang HY, Tien LT, Chen $\mathrm{SH}$, et al. Neuropeptide $\mathrm{Y}$ modulates c-Fos protein expression in the cuneate nucleus and contributes to mechanical hypersensitivity following rat median nerve injury. Journal of neurotrauma. 2009;26(9):1609-21.
50. Fostel JM. Future of toxicogenomics and safety signatures: balancing public access to data with proprietary drug discovery. Pharmacogenomics. 2007;8(5):425-30.

51. Mattingly CJ, Rosenstein MC, Colby GT, Forrest J $\mathrm{N}$, Boyer JL. The comparative toxicogenomics database (CTD): a resource for comparative toxicological studies. J Exp Zool A Comp Exp Biol. 2006;305:689-92.

52. Ellinger-Ziegelbauer $\mathrm{H}$, Aubrecht J, Kleinjans JC, Ahr HJ. Application of toxicogenomics to study mechanisms of genotoxicity and carcinogenicity. Toxicol Lett. 2009;186:36-44.

53. Sawada H, Takami K, Asahi S. A toxicogenomic approach to drug-induced phospholipidosis: analysis of its induction mechanism and establishment of a novel in vitro screening system. Toxicol Sci. 2001;83:282-92.

54. Cai D, Deng K, Mellado W, Lee J, Ratan RR, Filbin MT. Arginase I and polyamines act downstream from cyclic AMP in overcoming inhibition of axonal growth MAG and myelin in vitro. Neuron. 2002;35(4):711-9.

55. Judson RS, Richard AM, Dix DJ, Houck K, Martin MT, Kavlock RJ, et al. The toxicity data landscape for environmental chemicals. Environ Health Perspect. 2009;117:685-95.

56. Kavlock RJ, Austin CP, Tice RR. Toxicity testing in the $21^{\text {st }}$ century: implications for human health risk assessment. Risk Anal. 2009;29:485-7.

57. Ruepp SU, Tonge RP, Shaw J, Wallis N, Pognan F. Genomics and proteomics analysis of acetaminophen toxicity in mouse liver. Toxicol Sci. 2002;65:135-50.

58. Hogstrand C, Balesaria S, Glover CN. Application of genomics and proteomics for study of the integrated response to zinc exposure in a non-model fish species, the rainbow trout. Comp Biochem Physiol B. 2002;133:523-35.

59. Maine EM. Studying gene function in caenorhabditis elegans using RNA-mediated interference. Brief Funct. Genomic Proteomic. 2008;7:184-94.

60. Juan HF. Biomic study of human meyloid leukemia cells differentiation to macrophages using DNA array, proteomic, and bioinformatic analytical methods. Electrophoresis. 2002;23:2490-504.

61. Thomas RS, Pluta L, Yang L, Halsey TA. Application of genomic biomarkers to predict increased lung tumor incidence in 2-year rodent cancer bioassays. Toxicol Sci. 2007;97:55-64.

62. Hayes KR, Vollrath AL, Zastrow GM, McMillan BJ, Craven M, Jovanovich S, Rank DR, et al. EDGE: a centralized resource for the comparison, analysis, and distribution of toxicogenomic information. Mol. Pharmacol. 2005;67:1360-8.

63. Lindon JC. Contemporary issues in toxicology the role of metabonomics in toxicology and its evaluation by the COMET project. Toxicol Appl Pharmacol. 2003;187:137-46. 
64. Amin RP. Genomic interrogation of mechanism(s) underlying cellular responses to toxicants. Toxicology. 2002;181-2:555-63.

65. Weinshilboum RM, Wang L. Pharmacogenetics and pharmacogenomics: development, science, and translation. Annu Rev Genomics Hum Genet. 2006;7:223-45.

66. Amin RP, Vickers AE, Sistare F, Thompson KL, Roman RJ, Lawton M, et al. Identification of putative gene-based markers of renal toxicity. Environ Health Perspect. 2004;112:465-79.

67. Kita Y. Implications of circadian gene expression in kidney, liver and the effects of fasting on pharmacogenomic studies. Pharmacogenetics. 2002;12:55-65.

68. Yang C, Arnby CH, Arvidson K, Aveston S, Benigni $\mathrm{R}$, Benz $\mathrm{RD}$, et al. Understanding genetic toxicity through data mining: The process of building knowledge by integrating multiple genetic toxicity databases. Tox Mech Meth. 2008;18:277-95.

69. Kramer JA, Pettit SD, Amin RP, Bertram TA, Car B, Cunningham $\mathrm{M}$, et al. Overview of the application of transcription profiling using selected nephrotoxicants for toxicology assessment. Environ Health Perspect. 2004;112:460-4.

70. Bowtell DD. Options available-from start to finishfor obtaining expression data by microarray. Nat Genet. 1999;21(Suppl. 1):25-32.

71. Spellman PT, Miller M, Stewart J. Design and implementation of microarray gene expression markup language (MAGE-ML). Genome Biol. 2002;3(9):46.

72. Cunningham ML, Lehman-McKeeman L. Applying toxicogenomics in mechanistic and predictive toxicology. Toxicol Sci. 2005;83:205-6.

73. Wittliff JL, Erlander MG. Laser capture microdissection and its applications in genomics and proteomics. Methods Enzymol. 2002;356:12-25.

74. Kimura J, Nguyen ST, Liu H, Taira N, Miki Y, Yoshida K. A functional genome-wide RNAi screen identifies TAF1 as a regulator for apoptosis in response to genotoxic stress. Nucleic Acids Res. 2008;36:5250-9.

75. Harries HM, Fletcher ST, Duggan CM, Baker VA. The use of genomics technology to investigate gene expression changes in cultured human liver cells. Toxicol In Vitro. 2001;15(4-5):399-405.

76. Emmert-Buck MR. Laser capture microdissection. Science. 1996;274:998-1001.

77. Jain KK. Application of laser capture microdissection to proteomics. Methods Enzymol. 2002;356:157-67.

78. Karsten SL, Van Deerlin VM, Sabatti C, Gill LH, Geschwind DH. An evaluation of tyramide signal amplification and archived fixed and frozen tissue in microarray gene expression analysis. Nucleic Acids Res. 2002;30:E4.

79. Bonner RF. Laser capture microdissection: molecular analysis of tissue. Science. 1997;278:1481-3.
80. Venter JC, Adams MD, Myers EW. The sequence of the human genome. Science. 2011;291(5507):130451 .

81. Wolfinger RD, Gibson G, Wolfinger ED, Bennett L, Hamadeh H, Bushel $P$, et al. Assessing gene significance from cDNA microarray expression data via mixed models. J Comput Biol. 2001;8:625-37.

82. Quackenbush J. Computational analysis of microarray data. Nature Rev Genet. 2001;2:418-27.

83. Farland WH. Cancer risk assessment: evolution of the process. Prev Med. 1996;25:24-5.

84. Larsen JC, Farland W, Winters D. Current risk assessment approaches in different countries. Food Addit Contam. 2000;17:359-69.

85. Barry WT, Nobel AB, Wright FA. Significance analysis of functional categories in gene expression studies: a structured permutation approach. Bioinformatics. 2005;21(9):1943-9.

86. Ideker T, Galitski T, Hood L. A new approach to decoding life: systems biology. Annu Rev Genomics Hum Genet. 2001;2:343-72.

87. Xirasagar S, Gustafson SF, Huang CC, Pan Q, Fostel $\mathrm{J}$, Boyer $\mathrm{P}$, et al. Chemical effects in biological systems (CEBS) object model for toxicology data, SysTox-OM: design and application. Bioinformatics 2006;22:874-82.

88. Jeong SY, Park HJ, Oh JH, Kim CY, Yoon SJ. Gene expression profiling of doxifluridine treated liver, small and large intestine in cynomolgus (macaca fascicularis) monkeys. Molecularand Cellular Toxicology. 2007;3:137-44.

89. Wang EJ, Snyder RD, Fielden MR, Smith RJ, Gu YZ. Validation of putative genomic biomarkers of nephrotoxicity in rats. Toxicology. 2008;246:91-100.

90. Begley TJ, Rosenbach AS, Ideker T, Samson LD. Damage recovery pathways in Saccharomyces cerevisiae revealed by genomic phenotyping and interactome mapping. Mol Cancer Res. 2002;1:10312.

91. Kim YJ, Song MK, Song M, Ryu JC. Toxicogenomic study to identify potential new mechanistic markers on direct-acting mutagens in human hepatocytes (THLE-3). Molecular and Cellular Toxicology. 2007;3:231-7.

92. Lewis F, Maughan NJ, Smith V, Hillan K, Quirke P. Unlocking the archive-gene expression in paraffinembedded tissue. J Pathol. 2001;195:66-71.

93. Farland WH. The U.S. environmental protection agency's risk assessment guidelines: current status and future directions. Toxicol Ind Health 1992;8:20512.

94. Wetmore BA, Merrick BA. Toxicoproteomics: proteomics applied to toxicology and pathology. Toxicol Pathol. 2004;32:619-42.

95. Stuart GW, Berry MW. A comprehensive whole genome bacterial phylogeny using correlated peptide motifs defined in a high dimensional vector space. $\mathrm{J}$ Bioinform Comput Biol. 2003;1:475-93. 
96. Kazius J, McGuire R, Bursi R. Derivation and validation of toxicophores for mutagenicity prediction. J Med Chem. 2005;48:312-20.

97. Waring JF, Cavet G, Jolly RA, McDowell J, Dai H, Ciurlionis R, et al. Development of a DNA microarray for oxicology based on hepatotoxinregulate sequences. Environ Health Perspect. 2003;111:863-70.

98. Prelic A, Bleuler S, Zimmermann P, Wille A, Buhlmann P, Gruissem W, et al. A systematic comparison and evaluation of biclustering methods for gene expression data. Bioinformatics. 2006;22:1122-9.
99. Foster WR, Chen SJ, He A, Truong A, Bhaskaran V, Nelson DM, et al. A retrospective analysis of toxicogenomics in the safety assessment of drug candidates. Toxicol Pathol. 2007;35:621-35.

100.Schena M, Shalon D, Davis RW, Brown PO. Quantitative monitoring of gene expression patterns with a complementary DNA microarray. Science. 1995;270:467-70.

101.Watson JD, Crick FH. Molecular structure of nucleic acids; a structure for deoxyribose nucleic acid. Nature. 1953;171:737-8.

Cite this article as: Mahmud SMN, Mahmud S, Tandra TT, Kar A, Shathy EJ, Jahan N. The advent of system toxicology: aims and aspect of toxicogenomics. Int J Basic Clin Pharmacol 2016;5:1164-74. 